\title{
The influences of extraction time and pressure on the chemical characteristics of Gyejibokryeong-hwan decoctions
}

\author{
Jung-Hoon Kim ${ }^{1,2 \#}$, Nari Lee ${ }^{1}$, Hyeun-Kyoo Shin ${ }^{1}$, Chang-Seob Seo ${ }^{1 *}$ \\ 1 : Herbal Medicine Formulation Research Group, Korea Institute of Oriental Medicine \\ 2 : Division of Pharmacology, School of Korean Medicine, Pusan National University
}

\begin{abstract}
Objectives : This study was aimed to compare Gyejibokryeong-hwan (GBH) decoctions produced using different pressure levels for various extraction times to find the optimal extraction conditions through extraction yield, total soluble solids content (TSSC), hydrogen ion concentration $(\mathrm{pH})$, and the contents of chemical compounds.

Methods : Decoctions of GBH were prepared under the pressure levels of 0 or $1 \mathrm{kgf} / \mathrm{cm}^{2}$ for 30-180 min using water as extraction solvent. The extraction yield, TSSC, and $\mathrm{pH}$ were measured, and the amounts of the chemical compounds were determined using high performance liquid chromatography-photodiode array detector.

Results : The higher pressure and longer extraction time increased the values of TSSC and extraction yield, while decreased the $\mathrm{pH}$ value. The decoctions produced in $180 \mathrm{~min}$ by pressurized method and produced in 150 min by non-pressurized method showed maximum values of extraction yield and TSSC with minimum value of $\mathrm{pH}$. The amounts of chemical compounds showed variations in pressurized and non-pressurized decoction during overall extraction times. The influences of pressure and extraction time on extraction yield, TSSC, $\mathrm{pH}$, and the contents of chemical compounds were confirmed by regression analysis, which showed that all extraction values were significantly affected by at least one of two extraction factors, pressure and extraction time.

Conclusions : This study suggests that the pressure and extraction time can significantly affect the extraction efficiency of components from GBH decoctions. However, optimal extraction conditions could not be chosen due to the variation of the amounts of chemical compounds.
\end{abstract}

Key words : Gyejibokryeong-hwan, extraction factor, extraction yield, total soluble solids content, chemical compounds, regression analysis

\section{Introduction}

Gyejibokryeong-hwan (GBH), a traditional herbal formula, consists of five herbal medicines, including the twig of Cinnamomum cassia (Nees \& T. Nees) J. Presl, the sclerotium of Poria cocos F.A. Wolf, the root bark of Paeonia suffruticosa Andrews, the root of Paeonia lactiflora Pall., and the seed of Prunus persica Batsch. GBH has been clinically used to treat gynaecological diseases such as uterine myoma, hysteromyoma, and hot flushes ${ }^{1-3)}$.
Decoction is the extract which is prepared by heating the mixture of herbal medicines with water and is most used dosage form of herbal formula because of its convenient and rapid administration. As decoction is produced by heating the materials, various extraction factors, such as temperature, extraction time, solvent type, or pressure, can affect the extraction efficiency of constituents from the herbal medicines. Generally, higher pressure and longer extraction time can produce elevated amounts of chemical components from herbal

\footnotetext{
*Corresponding author : Chang-Seob, Seo. Herbal Medicine Formulation Research Group, Korea Institute of Oriental Medicine, Daejeon, Republic of Korea, 305-811

· Tel : +82-42-868-9361 · E-mail : csseo0914@kiom.re.kr

\#First author : Jung-Hoon, Kim. Herbal Medicine Formulation Research Group, Korea Institute of Oriental Medicine, Daejeon, Republic of Korea, 305-811

Present address of the first author : Division of Pharmacology, School of Korean Medicine, Pusan National University, Yangsan-si, Gyeongnam, Republic of Korea, 626-870

· Tel : +82-51-510-8456 ·E-mail : kmsct@pusan.ac.kr

- Received : 16 October 2014 - Revised : 27 October 2014 - Accepted : 28 October 2014
} 
medicine $e^{4,5)}$. Finding optimal extraction conditions by adjusting extraction factors such extraction time, temperature, or pressure have been issued ${ }^{6-8)}$. However, the influence of those extraction factors, including pressure or extraction time, on the changes of components in herbal decoction has not been investigated.

In the present study, the GBH decoctions were prepared by using pressurized $\left(1 \mathrm{kgf} / \mathrm{cm}^{2}\right)$ or non-pressurized condition $\left(0 \mathrm{kgf} / \mathrm{cm}^{2}\right)$ for extraction times from $30 \mathrm{~min}$ to $180 \mathrm{~min}$ with extraction temperature maintained at $100{ }^{\circ} \mathrm{C}$. The extraction yield, total soluble solids content (TSSC), and hydrogen ion concentration ( $\mathrm{pH})$ were compared. The quantification of $\mathrm{GBH}$ decoction was performed using high performance liquid chromatography coupled with photodiode array detector and the contents of chemical compounds were also compared. The influence of extraction factors on extraction variables, such as extraction yield, TSSC, $\mathrm{pH}$, or the content of each chemical compound in pressurized and non-pressurized decoction was investigated using regression analysis.

\section{Materials and Methods}

\section{Reagents and herbal materials}

HPLC-grade methanol, acetonitrile, and water were purchased from J.T. Baker Inc. (Phillipsburg, NJ, USA). Gallic acid, amygdalin, benzoic acid, coumarin, and cinnamic acid were purchased from Sigma-Aldrich (St Louis, MO, USA). Oxypaeoniflorin and benzoylpaeoniflorin were purchased from Chengdu Biopurify Phytochemicals Ltd. (Chengdu, China). Albiflorin, paeoniflorin, cinnamaldehyde, and paeonol were obtained from Wako Pure Chemical Industries Ltd. (Osaka, Japan). The purity of all marker compounds was $>98 \%$ and their chemical structures are shown in Fig. 1.

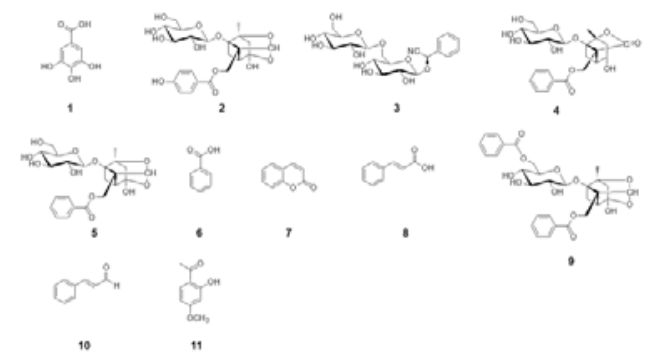

Fig. 1. Chemical structures of 11 standard compounds in Gyejibokryeong-hwan (GBH). 1: Gallic acid, 2: oxypaeoniflorin, 3: amygdalin, 4: albiflorin, 5: paeoniflorin, 6: benzoic acid, 7: coumarin, 8: cinnamic acid, 9: benzoylpaeoniflorin, 10: cinnamaldehyde, 11: paeonol.

The herbal medicines were purchased from the herbal medicine company Kwangmyungdang Medicinal Herbs (Ulsan, Korea). A voucher specimen (2014-KE31-1-5) has been deposited in the Herbal Medicine Formulation Research Group of the Korea Institute of Oriental Medicine. Herbal composition of $\mathrm{GBH}$ is shown in Table 1.

Table 1. Composition of herbal medicines of Gyejibokryeong-hwan $(\mathrm{GBH})$

\begin{tabular}{llc}
\hline \multicolumn{1}{c}{ Herbal medicine } & \multicolumn{1}{c}{ Original region } & Amount $(\mathrm{g})$ \\
\hline \hline Cinnamomum cassia (Nees \& T. & Vietnam & 3.75 \\
Nees) J. Presl & Gangwon, Korea & 3.75 \\
Poria cocos F.A. Wolf & Chungbuk, Korea & 3.75 \\
Paeonia suffruticosa Andrews & Gyeongbuk, Korea & 3.75 \\
Paeonia lactiflora Pall. & South Africa & 3.75 \\
Prunus persica Batsch & & \\
\hline
\end{tabular}

\section{Preparation of standard solutions}

Accurately weighed standard compounds were dissolved in methanol to produce stock solutions at concentrations of $1 \mathrm{mg} / \mathrm{mL}$. Each stock solution was diluted to make working solutions, which were used to construct calibration curves.

\section{Preparation of GBH decoctions and samples}

The mixture of herbal medicines composing $\mathrm{GBH}$ (750 g corresponding to double amount of one formula set, 'Je' in Korean) were extracted at $100{ }^{\circ} \mathrm{C}$ in water using a high-speed vacuum herb extractor (Cosmos 660, Kyungseo Machine, Incheon, Korea). The extraction was carried out under pressurized $\left(1.0 \mathrm{kgf} / \mathrm{cm}^{2}\right)$ or non-pressurized $\left(0 \mathrm{kgf} / \mathrm{cm}^{2}\right)$ methods for 30, 60, 90, 120, 150, and $180 \mathrm{~min}$. The extraction water was regulated to make the final volumes of the decoctions around $3800 \mathrm{~mL}$. A $50 \mathrm{~mL}$ of each decoction was lyophilized using a freeze-drier (IlshinBioBase, Dongducheon, Korea) to make a powder, which was then dissolved in HPLC-grade water and was filtered through a 0.2 $\mathrm{mm}$ syringe filter (SmartPor ${ }^{\circledR}$; Woongki Science, Korea) prior to HPLC injection.

\section{Measurements of extraction yield, total} soluble solids content (TSSC), and hydrogen ion concentration $(\mathrm{pH})$

To calculate the yield of decoctions, the weight of each freeze-dried decoction was converted to a percentage of the formula used for a single extraction. TSSC ( ${ }^{\circ}$ Brix) of each decoction was measured using a refractometer (Pal-a; ATAGO, Tokyo, Japan). A pH was determined with a $\mathrm{pH}$ meter (672 pH/Ion meter; Metrohm, Switzerland). 


\section{Chromatographic conditions}

The HPLC-PDA system comprised a Shimadzu LC-20A (Shimadzu Corporation, Kyoto, Japan) equipped with a solvent delivery unit (LC-20AT), autosampler (SIL-20AC), column oven (CTO-20A), degasser (DGU-20A A $_{3}$, and PDA (SPD-M20A). The acquired data were processed using LabSolutions software (Ver. 5.3; Shimadzu, Japan). Separation was performed on a Gemini $\mathrm{C}_{18}$ column $(4.6 \times 250 \mathrm{~mm}, 5 \mu \mathrm{m}$; Phenomenex, Torrance, CA, USA) maintained at $40{ }^{\circ} \mathrm{C}$. The flow rate was 1.0 $\mathrm{mL} / \mathrm{min}$ and the injection volume was set to $10 \mu \mathrm{L}$. The mobile phase consisted of water (A) and acetonitrile (B), both containing 1\% acetic acid. Gradient elution of the mobile phase was applied: 10-60\% (B) over 0$30 \mathrm{~min}, 60-100 \%$ (B) over 30-40 min, held for $5 \mathrm{~min}$ and then re-equilibrated to $10 \%$ until the end of the analysis. The detection wavelength for each compound was screened from $190 \mathrm{~nm}$ to $400 \mathrm{~nm}$ and optimal wavelength was set according to the maximum absorption wavelengths of the standard compounds (230, 260, 270 and $275 \mathrm{~nm}$ ). The analytical conditions in the previous work of our laboratory were applied to this study (Kim JH et al. Arch Pharm Res. 2014).

\section{Statistical analyses}

All experiments were performed in triplicate. Two-tailed t-tests and Dunnett's test were conducted for the two-group and the multi-group comparisons using Microsoft Excel (Microsoft, Redmond, WA, USA) and SYSTAT 10 (SPSS Inc., Chicago, IL, USA). Differences were considered significant at $\mathrm{P}<0.05, \mathrm{P}<0.01$, or $\mathrm{P}<0.001$. Regression analysis was performed through extraction yields, TSSC, $\mathrm{pH}$, and the amount of each marker compound using open source software 'R (ver. 3.0.2)'.

\section{Results}

\section{Comparison of extraction yield, TSSC, and}

\section{$\mathrm{pH}$ in $\mathrm{GBH}$ decoctions}

The decoction produced by pressurized method showed higher extraction yield as extraction time increased, however, the pressure did not showed significant difference of extraction yields in all time courses (Fig. 2A). The results of TSSC showed similar pattern with that of extraction yield. TSSC was significantly increased with increased extraction time in both by pressurized and non-pressurized decoctions. Moreover, significantly higher TSSC value was observed in the pressurized decoctions in 90 and $180 \mathrm{~min}$ (Fig. 2B). There was significant difference of the $\mathrm{pH}$ between the pressurized and non-pressurized decoctions after initial extraction time, 30 min (Fig. 2C).
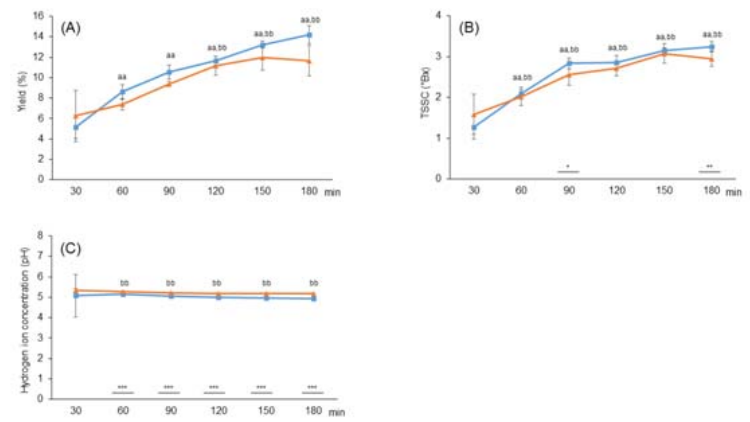

Fig. 2. Variation of extraction yield (A), total soluble solids content (B), and hydrogen ion concentration (C) in GBH decoctions produced by pressurized $(\boldsymbol{\square})$ and non-pressurized $(\boldsymbol{\Lambda})$ extraction methods for extraction time. Data was expressed as average of triplicate measurements. Statistically significant at ${ }^{*} P\left\langle 0.05,{ }^{* *} P<\right.$ 0.01 or ${ }^{* * *} P<0.001$ of difference in values between pressurized and non-pressurized extraction methods. ${ }^{a} P<0.05,{ }^{a a} P<0.01$, and ${ }^{\text {aad }} P<0.001$ versus the decoction produced by pressurized method at 30 min. ${ }^{b} P<0.05,{ }^{b b} P<0.01$, and ${ }^{b b b} P<0.001$ versus the decoction produced by non-pressurized method at $30 \mathrm{~min}$.

\section{Comparison of the contents of the marker} compounds in GBH decoctions

Linear equation, correlation coefficients $\left(r^{2}\right)$, limit of detection, and limit of quantification in previous paper of our laboratory (Kim JH et al. Arch Pharm Res. 2014) were applied to this study as shown in Table $2^{1)}$. The eleven marker compounds, gallic acid, oxypaeoniflorin, amygdalin, albiflorin, paeoniflorin, benzoic acid, coumarin, cinnamic acid, benzoylpaeoniflorin, cinnamaldehyde, and paeonol, detected in the GBH decoction were well separated on chromatograms without overlapping or interruption by adjacent peak by using the methods described above (Fig. 3 and 4).

Table 2: Linear equation, correlation coefficients $\left(r^{2}\right), L O D$, and $\mathrm{LOQ}$ for the bioactive compounds in $\mathrm{GBH}$

\begin{tabular}{llcccc}
\hline \multicolumn{1}{c}{ Compound } & \multicolumn{1}{c}{ Linear equation } & $\mathrm{r}^{2}$ & $\begin{array}{c}\text { Linear range } \\
(\mathrm{mg} / \mathrm{mL})\end{array}$ & $\begin{array}{c}\mathrm{LOD} \\
(\mathrm{mg} / \mathrm{mL})\end{array}$ & $\begin{array}{c}\mathrm{LOQ} \\
(\mathrm{mg} / \mathrm{mL})\end{array}$ \\
\hline \hline Gallic acid & $\mathrm{y}=31871 \mathrm{x}-8708.6$ & 0.9999 & $3.13-50.00$ & 0.03 & 0.13 \\
Oxypaeoniflorin & $\mathrm{y}=11603 \mathrm{x}-4882.1$ & 0.9994 & $1.56-25.00$ & 0.08 & 0.30 \\
Amygdalin & $\mathrm{y}=308.43 \mathrm{x}+48.26$ & 1.0000 & $3.52-225.00$ & 2.11 & 7.04 \\
Albiflorin & $\mathrm{y}=9522.33 \mathrm{x}-1496.19$ & 1.0000 & $0.39-50.00$ & 0.16 & 0.52 \\
Paeoniflorin & $\mathrm{y}=8911.69 \mathrm{x}-6277.17$ & 1.0000 & $2.34-300.00$ & 0.47 & 1.56 \\
Benzoic acid & $\mathrm{y}=2458.9 \mathrm{x}+30.667$ & 1.0000 & $1.56-25.00$ & 0.38 & 1.36 \\
Coumarin & $\mathrm{y}=48149.55 \mathrm{x}+2606$ & 1.0000 & $0.39-50.00$ & 0.04 & 0.13 \\
Cinnamic acid & $\mathrm{y}=89229.20 \mathrm{x}+812.34$ & 1.0000 & $0.08-10.00$ & 0.02 & 0.06 \\
Benzoylpaeoniflorin & $\mathrm{y}=1685.60 \mathrm{x}-127.00$ & 1.0000 & $2.50-10.00$ & 0.56 & 1.86 \\
Cinnamaldehyde & $\mathrm{y}=112104.36 \mathrm{x}+4133.03$ & 1.0000 & $0.31-40.00$ & 0.02 & 0.06 \\
Paeonol & $\mathrm{y}=63861 \mathrm{x}+23842.04$ & 0.9999 & $0.78-100.00$ & 0.03 & 0.10 \\
\hline
\end{tabular}

LOD, limit of detection; $L O Q$, limit of quantification

$\mathrm{y}$, peak area $(\mathrm{mAU}) ; \mathrm{x}$, concentration of compound $(\mu \mathrm{g} / \mathrm{mL})$.

Linear equation, correlation coefficients $\left(r^{2}\right), \mathrm{LOD}$, and $\mathrm{LOQ}$ in previous study (Kim et al. Arch Pharm Res. 2014) were applied. 


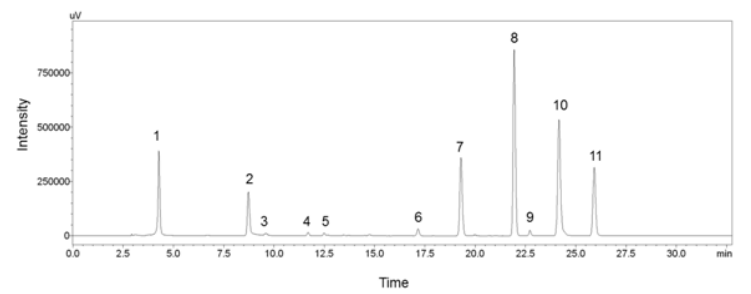

Fig. 3. Chromatogram of standard compound in GBH. 1: Gallic acid, 2: oxypaeoniflorin, 3: amygdalin, 4: albiflorin, 5: paeoniflorin, 6: benzoic acid, 7: coumarin, 8: cinnamic acid, 9: benzoylpaeoniflorin, 10: cinnamaldehyde, 11: paeonol.

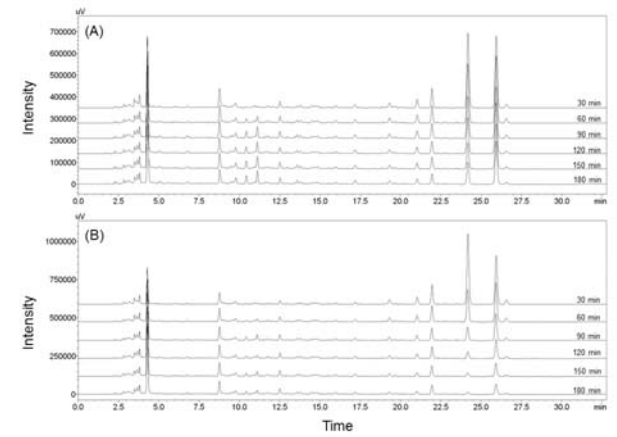

Fig 4. Representative chromatograms of $\mathrm{GBH}$ decoctions produced by pressurized method (A) and non-pressurized method (B) at UV $260 \mathrm{~nm}$.

Pressurized method produced significantly higher content of gallic acid and benzoic acid in most extraction time and the contents of two compounds were increased (slightly in the content of benzoic acid), as the extraction time increased in both pressurized and non-pressurized decoctions. The contents of oxypaeoniflorin and paeoniflorin were significantly different between both decoctions in all time courses and their differences were larger as extraction time increased: the contents of oxypaeoniflorin and paeoniflorin in non-pressurized decoctions were increased, while the contents of those compounds in pressurized decoctions were decreased. Amygdalin and benzoylpaeoniflorin, which also showed higher amounts in non-pressurized decoctions, were decreasingly extracted in both decoctions over peak amount time, 60 or $90 \mathrm{~min}$. The contents of coumarin and cinnamic acid were decreased with extraction time increased, and the difference of contents between pressurized and non-pressurized decoctions were significant in extraction times. In contrast, the contents of cinnamaldehyde and paeonol were steeply decreased in the decoction produced by non-pressurized method after initial time of extraction. The contents of those two compounds in pressurized decoction, which were higher than in non-pressurized decoction, were also decreased with increased extraction time (Fig. 5).

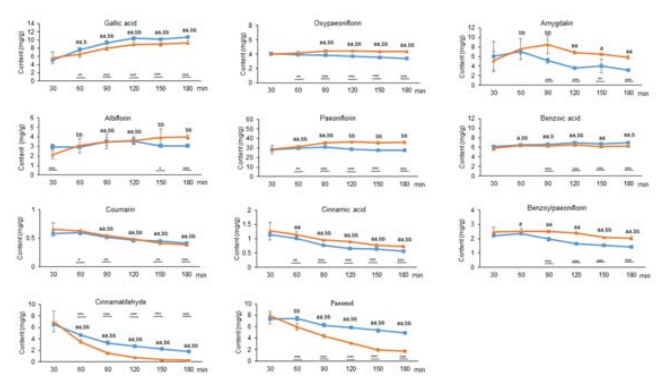

Fig. 5. Variation of the content of 11 marker compounds in GBH decoctions produced by pressurized $(\boldsymbol{\square})$ and non-pressurized $(\boldsymbol{\Lambda})$ extraction methods for extraction time. Data was expressed as average of triplicate measurements. Statistically significant at ${ }^{*} P<$ $0.05, P<0.01$ or ${ }^{* * * x} P<0.001$ of difference in values between pressurized and non-pressurized extraction methods. ${ }^{a} P<0.05,{ }^{a a} P<0.01$, and ${ }^{a a a} P<0.001$ versus the decoction produced by pressurized method at 30 min. ${ }^{b} P<0.05,{ }^{b b} P<0.01$, and ${ }^{b b b} P<0.001$ versus the decoction produced by non-pressurized method at $30 \mathrm{~min}$.

\section{Regression analysis of the influence of the pressure and extraction on extraction yield, TSSC,} $\mathrm{pH}$, and the content of each marker compound

Regression analysis was carried out to investigate the influence of extraction factors (pressure and extraction time) on the variables (extraction yield, TSSC, $\mathrm{pH}$, and the content of each compound). The adjusted regression coefficients $\left(\mathrm{R}_{\text {adj }}^{2}\right)$ of extraction yield, TSSC, and $\mathrm{pH}$ were more than 0.8 with significant $F$ - and $p$-values. The extraction time significantly influenced to extraction yield, TSSC, and $\mathrm{pH}$, while pressure showed significant influence only on the hydrogen ion concentration. The $\mathrm{R}_{\text {adj }}^{2}$ of the contents of 11 marker compounds were 0.30 $<r^{2}<0.95$ with significant $p$-values, except for albiflorin with $p>0.05$. Pressure and extraction time both significantly influenced to the contents of benzoic acid, cinnamic acid, benzoylpaeoniflorin, and paeonol, while the contents of the rest compounds were affected by at least one of two extraction factors, pressure or extraction time (Table 3).

Table 3: Regression analysis of extraction factors (pressure and extraction time) on variables

\begin{tabular}{|c|c|c|c|c|c|c|}
\hline \multirow{2}{*}{ Variable } & \multicolumn{2}{|c|}{$p$-values of coefficients } & \multirow{2}{*}{$\mathrm{DF}$} & \multirow{2}{*}{$\mathrm{R}_{\mathrm{adj}}^{2}$} & \multirow{2}{*}{ F-value } & \multirow{2}{*}{$p$-value } \\
\hline & Pressure & Extraction time & & & & \\
\hline Extraction yield & $>0.05$ & $\langle 0.001$ & 9 & 0.8922 & 46.53 & $\langle 0.001$ \\
\hline Total soluble solids content & $>0.05$ & $<0.001$ & 9 & 0.8201 & 26.08 & $<0.001$ \\
\hline Hydrogen ion concentration & $<0.001$ & $<0.001$ & 9 & 0.9115 & 57.63 & $<0.001$ \\
\hline \multicolumn{7}{|l|}{ The content of compound } \\
\hline Gallic acid & $>0.05$ & $<0.001$ & 9 & 0.8111 & 24.62 & $<0.001$ \\
\hline Oxypaeoniflorin & $<0.01$ & $>0.05$ & 9 & 0.6205 & 9.993 & $<0.01$ \\
\hline Amygdalin & $<0.05$ & $>0.05$ & 9 & 0.4323 & 5.188 & $<0.05$ \\
\hline Albiflorin & $>0.05$ & $<0.05$ & 9 & 0.3129 & 3.505 & $>0.05$ \\
\hline Paeoniflorin & $<0.01$ & $>0.05$ & 9 & 0.5711 & 8.323 & $<0.01$ \\
\hline Benzoic acid & $<0.01$ & $<0.05$ & 9 & 0.6616 & 11.75 & $<0.01$ \\
\hline Coumarin & $>0.05$ & $<0.001$ & 9 & 0.9018 & 51.48 & $<0.001$ \\
\hline Cinnamic acid & $<0.001$ & $<0.001$ & 9 & 0.9378 & 83.91 & $<0.001$ \\
\hline Benzoylpaeoniflorin & $<0.001$ & $<0.001$ & 9 & 0.8595 & 34.65 & $<0.001$ \\
\hline Cinnamaldehyde & $>0.05$ & $<0.001$ & 9 & 0.7899 & 21.68 & $<0.001$ \\
\hline Paeonol & $<0.01$ & $<0.001$ & 9 & 0.8370 & 29.25 & $<0.001$ \\
\hline
\end{tabular}




\section{Discussions}

In the present study, the influences of extraction factors (pressure and extraction time) to variables such as extraction yield, total soluble solids content (TSSC), hydrogen ion concentration $(\mathrm{pH})$, and the content of chemical compounds were investigated in GBH decoctions.

Extraction yield and TSSC, which consist of plant cell ingredients released when plant cell wall is broken down, were apparently affected by pressure and extraction time. Higher pressure and longer extraction time can increase the extraction efficiency of ingredients from plant cell, and thus extraction yield and TSSC are enchanced ${ }^{9,10)}$. These results indicate that the power of solvent is increased at higher pressure and longer extraction time, which makes plant tissue cell wall easily broken down, and mass transfer rate between solvent and ingredients in the cell is elevated. This can improve the cell permeability and secondary metabolite diffusion to solvent ${ }^{11-13)}$. Higher extraction pressure can also reduce the $\mathrm{pH}$ of solvent during extraction, because higher phenolics are released into the solvent and molecules in the extracts are deprotonated, which can elevate the extraction of bioactive compounds stable at lower $\mathrm{pH}^{14)}$.

Contrary to the results from extraction yield, TSSC, and $\mathrm{pH}$, the content of marker compounds showed various patterns when influenced by pressure and extraction time. Higher pressure and longer extraction time produced more gallic acid and benzoic acid as pressurized extraction forms higher amounts of phenolic compounds ${ }^{11,15)}$. Other compounds, such as oxypaeoniflorin, amygdalin, paeoniflorin, cinnamic acid, and benzoylpaeoniflorin, were proportionally influenced by pressure and extraction time. The contents of those compounds were higher in non-pressurized decoctions and most of their values were decreased with extraction time increased, which indicates that some chemical compounds might be degraded or decomposed, or impurities which are unnecessary or toxic can be over-extracted from the herbal medicines in pressurized extraction condition with longer extraction time ${ }^{16)}$. Although the extraction time negatively influenced, the contents of two compounds, cinnamaldehyde and paeonol, were significantly lower in non-pressurized decoctions because of their volatile characteristics which can be easily evaporated along with solvent vapor when extracted in atmospheric conditions ${ }^{17,18)}$.

Regression analysis demonstrates that higher pressure and longer extraction time significantly affected 3 variables, extraction yield, TSSC, and contents of gallic acid and benzoic acid, showing the adjusted regression coefficients $\left(R_{\text {adj }}^{2}\right)>0.8$. These results indicate that model is significantly predictive under any combination of variables and extraction conditions which have positive effects on those variables ${ }^{19,20)}$. However, higher pressure and extraction time can negatively affect to $\mathrm{pH}$ and the extraction efficiency of several chemical compounds. Thus, further study is needed to find out optimal extraction condition of herbal formula, considering the effect of pressure and extraction time.

\section{Conclusions}

In the present study, we compared Gyejibokryeong-hwan (GBH) decoctions produced using different pressure levels (0 and $\left.1 \mathrm{kgf} / \mathrm{cm}^{2}\right)$

1. Higher pressure and longer extraction time positively affected the extract yield and the total soluble solid content (TSSC).

2. Higher pressure and longer extraction time negatively affected the hydrogen ion concentration $(\mathrm{pH})$.

3. The contents of chemical compounds showed various patterns according to pressure and extraction time.

We conclude that pressure and extraction time can influence the extraction efficiency of compositional constituents of GBH decoctions, however, in-depth study is necessary to find optimal extraction conditions for herbal formula, from the pharmacological viewpoint as well as the physicochemical viewpoint.

\section{Acknowledgements}

This work was supported by a grant from the Korea Institute of Oriental Medicine (K14030).

\section{References}

1. Sakamoto S, Yoshino H, Shirahata Y, Shimodairo K, Okamoto R. Pharmacotherapeutic effects of kuei-chih-fu-ling-wan (keishi-bukuryo-gan) on human uterine myomas. Am J Chin Med. 1992 ; 20(3-4) : 313-7.

2. Sang $H$. Clinical and experimental research into treatment of hysteromyoma with promoting qi flow and blood circulation, softening and resolving hard lump. J Traditi Chin Med. 2004 ; 24(4) : 274-9.

3. Ushiroyama T, Ikeda A, Sakuma K, Ueki M. Comparing the effects of estrogen and an herbal medicine on 
peripheral blood flow in post-menopausal women with hot flashes: hormone replacement therapy and gui-zhi-fu-ling-wan, a Kampo medicine. Am J Chin Med. 2005 ; 33(2) : 259-67.

4. Zhang S, Chen R, Wu H, Wang C. Ginsenoside extraction from Panax quinquefolium L. (American ginseng) root by using ultrahigh pressure. J Pharm Biomed Anal. 2006 ; 41(1) : 57-63.

5. Egizabal A, Zuloaga O, Etxebarria N, Fernández LA, Madariaga JM. Comparison of microwave-assisted extraction and Soxhlet extraction for phenols in soil samples using experimental designs. Analyst. 1998 ; $123: 1679-84$

6. Choi MP, Chan KK, Leung HW, Huie CW. Pressurized liquid extraction of active ingredients (ginsenosides) from medicinal plants using non-ionic surfactant solutions. J Chromatogr A. 2003 ; 983(1-2) : 153-62.

7. Eikani MH, Golmohammad F, Rowshanzamir S Subcritical water extraction of essential oils from coriander seeds (Coriandrum sativum L.). J Food Eng. 2007 ; 80(2) : 735-40.

8. Luthria DL. Influence of experimental conditions on the extraction of phenolic compounds from parsley (Petroselinum crispum) flakes using a pressurized liquid extractor. Food Chemistry. 2008 ; 107(2) : 745-52

9. Kaufmann B, Christen P. Recent extraction techniques for natural products: Microwave-assisted extraction and pressurised solvent extraction. Phytochem Anal. 2002 ; 13(2) : 105-13.

10. Kwak EJ. Development of brown colored rice tea with high GABA content. J Korean Soc Food Sci Nutr. 2010 ; 39(8) : 1201-5

11. Prasad KN, Yang B, Shi J, Yu C, Zhao M, Xue S, Jiang Y. Enhanced antioxidant and antityrosinase activities of longan fruit pericarp by ultra-high-pressure-assisted extraction. J Pharm Biomed Anal. 2010 ; 51(2) : 471-7.

12. Kim SH, Kim HK, Yang ES, Lee KY, Kim SD, Kim YC, Sung SH. Optimization of pressurized liquid extraction for spicatoside A in Liriope platyphylla. Sep Purif Technol. 2010 ; 71(2) : 168-72.

13. Corrales M, Toepfl S, Butz P, Knorr D, Tauscher B. Extraction of anthocyanins from grape by-products assisted by ultrasonics, high hydrostatic pressure or pulsed electric fields: A comparison. Inno Food Sci Emerg Technol. 2008 ; 9(1) : 85-91.

14. Prasad KN, Yang E, Yi C, Zhao M, Jiang Y. Effects of high pressure extraction on the extraction yield, total phenolic content and antioxidant activity of longan fruit pericarp. Innov Food Sci Emerg Technol. 2009 ; 10(2) : 155-9.

15. Ramamoorthy PK, Bono A. Antioxidant activity, total phenolic and flavonoid content of Morinda citrifolia fruit extracts from various extraction processes. J Eng Sci Technol. 2007 ; 2(1) : 70-80.

16. Rostagno MA, Palma M, Barroso CG. Ultrasound-assisted extraction of isoflavones from soy beverages blended with fruit juices. Anal Chim Acta. 2007 ; 597(2) : 265-72.

17. Chu K, Chen L, Xu Wei, Li H, Zhang Y, Xie W, Zheng J. Preparation of a paeonol-containing temperature-sensitive in situ gel and its preliminary efficacy on allergic rhinitis. Int J Mol Sci. 2013 ; 14(3) : 6499-515

18. Wang R, Wang R, Yang B. Extraction of essential oils from five cinnamon leaves and identification of their volatile compound compositions. Innov Food Sci Emerg Technol. 2009 ; 10(2) : 289-92.

19. Liu B, Guo F, Chang Y, Jiang H, Wang Q. Optimization of extraction of evodiamine and rutaecarpine from fruit of Evodia rutaecarpa using modified supercritical $\mathrm{CO}_{2}$. JChromatogr A. 2010 ; 1217(50) : 7833-9.

20. Zhong K, Wang Q. Optimization of ultrasonic extraction of polysaccharides from dried longan pulp using response surface methodology. Carbohydr Polym. 2010 ; 80(1) : 19-25. 